Ecologic Study of Nutritional Status of Ecuadorean Children

WILSON ORTIZ AND MARCO BORSOTTI Department of Pediatrics, Faculty of Medicine, Universidad Central del Ecuador, Universidad Tecnica de Ambato, Ecuador

The nutritional status of children is the product of many ecologic factors, including social, cultural, economic, educational, and emotional, as well as the biologic determinants: A descriptive study has been conducted to evaluate the relative importance of these variables to the nutritional status of rural and urban Ecuadorean children. Two hundred twenty-four families were evaluated. One hundred ninety-four of the families studied constitute the present investigation.

Significant differences were observed between urban and rural populations in terms of maternal age and maternal education. The average ingestion of animal proteins is much lower in the rural populations. Nutritional status is inferior in the rural children.

Multiple regression equations revealed a strong correlation between the nutritional status of the children and the ingestion of animal proteins. However, in specific population groups, a relationship was also evident between vegetable protein and nutritional status. Sociocultural environmental variables were significantly correlated with nutritional status; however, these variables differed in ranks of importance among the various rural and urban population groups.

It can be concluded that the nutritional status of the rural children is substantially less than that of the urban study group. The ecologic factors vary in relative importance among these study groups and appear to be specific in their impact on the nutritional status.

\section{Net Acid Balance in Marasmic Infants during Recovery from Diarrheal Dehydration}

G. SPEROTTO, F. R. CARRAZZA, AND A. L. CARDOSA Clinical Research Center of The Children's Institute, Hospital das Clinicas, Sao Paulo, Brazil

The net acid balance technique of Lennon et al. (1966) permits the simultaneous measurement of acid production and excretion. Seven marasmic infants were studied during the first 6 days of treatment for diarrheal dehydration. As a control, five well nourished infants were studied during recovery from dehydration.

Acid excretion was higher in marasmic infants, with significant differences on the third and sixth days. These differences are due to higher excretion of $\mathrm{NH}_{4}$ and titratable acidity. Acid production of marasmic infants was lower, with significant differences on the third and fourth days. These differences are due to lower excretion of $\mathrm{SO}_{4}$ and higher absorption of "combustible anions." Net acid balance was lower in marasmic infants with significant differences on the first and third days. Cumulated net acid balance was significantly lower in marasmic infants during all 6 days of study.

These findings suggest that, in marasmus, mechanisms of disposal of acid load are effective and are able to cope with acid loads generated during diarrheal episodes.

\section{Malnutrition and Adoption-Two Variables in Infant De- velopment}

M. CUSMINSKY, L. GARCIA, Z. DOPICH, E. CASTRO, M. C ALONSO, G. NARDUZZI, AND M. BENISSO Casa Cuna, La Plata, Province of Buenos Aires, Argentina

It is difficult to differentiate the effects of nutritional and psychologic factors during the recovery of malnourished children in the adoptive home. Our investigation attempted to differentiate the nutritional from psychologic stimuli in severely malnourished children who were institutionalized before being adopted by families. Twenty-six children were placed for adoption after hospital- ization in the Dr. N. H. Sbarra hospital. All of this study group had suffered from second or third degree malnutrition at the time of admission to the hospital. The mean age at hospitalization was 5 months and the mean stay in the hospital was 6 months. The physical and developmental progress was compared with a control group of 17 well nourished children of similar age at hospitalization and adoption. During the period of hospitalization, all infants received an individualized psychomotor stimulation program along with an adequate nutritional intake. Weight, supine length, language, and adaptive behaviour and developmental quotient were assessed at admission, at the time of adoption, and 6 and 12 months after adoption.

All malnourished infants demonstrated a rapid catch-up growth during nutritional rehabilitation in the hospital and during the first months after adoption. There was a significant reduction in rate of psychomotor development in both the malnourished and the control groups during hospitalization despite the stimulation plan. However, the effects of hospitalization on psychomotor development were no longer evident after 6 months of adoption. These observations indicate the independence of nutritional recovery from psychomotor development and confirm the importance of a stable family environment in the rehabilitation of infants.

\section{Chlordiazepoxide Effects on Learning Ability of Euthrophic and Marasmic Rats}

JOSE M. CELADON AND MARTHA COLUMBO Institute of Nutrition and Food Technology, Universidade de Chile, Santiago, Chile

The objective of this research is to demonstrate that emotion plays a role in learning of the undernourished rat. In other words, the high anxiety level of undernourished subjects acts negatively upon problem solving in different learning situations. Marasmus was induced in rats by increasing the litter size from birth until weaning. The reactivity and anxiety state of rats was modified using tranquilizer, chlordiazepoxide, $5 \mathrm{mg} / \mathrm{kg}$ body wt ip, administered $30 \mathrm{~min}$ before each test. Their response was evaluated in a Hebb-Williams maze.

The control group, well nourished rats receiving injections of $9 \% \mathrm{NaCl}$ committed $107 \pm 22.7$ errors in the 12 problems of the Hebb-Williams maze. This group showed the best performance. The well nourished group treated with chlordiazepoxide had a total error score of $196.9 \pm 42.2$. The marasmic group receiving $9 \% \mathrm{NaCl}$ injections committed $196.9 \pm 55.9$ errors, and the marasmic group with chlordiazepoxide, $148.56 \pm 33.4$ errors. Variance analysis gives an $\mathrm{F}=8.68$ which is significant $(P<$ 0.01 ). This result supported the hypothesis pointed out by Levitzky-Barnes that high anxiety level of undernourished subjects acts negatively upon problem solving. The marasmic group that received chlordiazepoxide injections committed less errors than the marasmic subjects injected with $9 \% \mathrm{NaCl}$. This difference was significant at a level of $P<0.01$.

\section{Balanced Translocation 46,t(13;18) (q21;q21) with Abnor- mal Phenotype: Possible Evidence for Position Effect in $18 q 21$}

ROBERTO COCO Laboratory of Cytogenetics, Division of Endocrinology, Hospital de Ninos, Buenos Aires, Argentina

Individuals with balanced chromosomal translocations generally do not have an abnormal phenotype, presumably because of unchanged content of genetic material. Thus, the finding of apparently balanced translocations in phenotypically abnormal individuals is surprising. This may be due to coincidence or may be related through some undetected chromosomal mechanism (small deletions or insertions at the breakpoints). However, even without chromosomal imbalance a translocation might produce an abnormal phenotype through a position effect. Position effects are known in other species, but the existence of this chromosomal 